\title{
Effectiveness and safety of oxaliplatin compared to cisplatin for advanced, unresectable gastric cancer: a systematic review and meta-analysis
}

\author{
Francesco Montagnani • Gina Turrisi • \\ Claudio Marinozzi · Camillo Aliberti • \\ Giammaria Fiorentini
}

Received: 10 August 2010/Accepted: 26 September 2010/Published online: 23 February 2011

(c) The International Gastric Cancer Association and The Japanese Gastric Cancer Association 2011

\begin{abstract}
Background Cisplatin has been largely used in the treatment of advanced, unresectable gastric cancer, mainly in combinations with fluoropyrimidines and anthracyclines. Oxaliplatin has been shown to be at least as effective as cisplatin for this disease, but with less toxicity and a better tolerability profile, especially for older patients. We performed a systematic review of the literature to address and quantify differences in the efficacy and the safety between oxaliplatin and cisplatin for the treatment of this disease.

Methods The literature was searched for randomized controlled trials (RCTs) comparing oxaliplatin to cisplatin. Odds ratios (ORs) with 95\% confidence intervals (CIs) were used to analyze dichotomous variables. Hazard ratios (HRs) for progression and death were combined with an inverse variance method based on logarithmic conversion. A fixed effect model and Mantel-Haenszel's (M-H) method were used. Heterogeneity was tested with the $Q$ test and the $I^{2}$ value. Sensitivity analyses were performed.

Results Three RCTs were identified, involving a total of 1294 patients. Oxaliplatin significantly improved progression-free survival $(\mathrm{HR}=0.88, p=0.02)$ and overall survival $(\mathrm{HR}=0.88, p=0.04)$. Moreover, it was associated with less neutropenia $(\mathrm{OR}=0.53, p<0.01)$ and fewer
\end{abstract}

F. Montagnani $(\varangle) \cdot$ G. Turrisi · G. Fiorentini Oncology Unit, Department of Medicine, S. Giuseppe Hospital, AUSL 11, Viale Boccaccio, 50053 Empoli, Florence, Italy e-mail: iago2003@libero.it

C. Marinozzi

Department of Oncology, AUSL 10, Florence, Italy

C. Aliberti

Interventional Radiology Unit, Department of Radiology,

Delta Hospital, AUSL, Ferrara, Italy thromboembolic events $(\mathrm{OR}=0.42, p<0.01)$, but it was also associated with increased neurotoxicity $(\mathrm{OR}=6.91$, $p<0.01$ ).

Conclusions Our results support the existence of a small but significant survival benefit of oxaliplatin over cisplatin. Oxaliplatin is associated with less toxicity and better tolerability, especially in older patients and when used in twodrug, bi-weekly regimens.

Keywords Oxaliplatin - Cisplatin - Advanced gastric cancer $\cdot$ Systematic review

\section{Introduction}

Adenocarcinoma of the stomach and esophagogastric junction (EGJ) represents the second leading cause of cancer-related death worldwide [1].

Surgery has been the mainstay of treatment for resectable disease and still represents the only curative treatment, either alone or combined with chemotherapy or chemoradiotherapy [2, 3]. Unfortunately a large majority of patients experience a relapse or present with unresectable locally advanced or metastatic disease.

Systemic chemotherapy is the only effective treatment available in these situations, but it has extremely poor results and the median survival time is invariably shorter than 1 year [4-6].

Multiple chemotherapeutic combinations have been developed in the past 40 years, mainly based on 5-fluorouracil (5-FU), cisplatin, anthracyclines, and taxanes.

A meta-analysis based on aggregate data showed that three-drug combinations conferred longer survival compared to monochemotherapy and two-drug combinations. However, the benefit is small and happens at the cost of 
increased toxicity [4]. Another platinum compound, oxaliplatin, could be a candidate to replace cisplatin, possibly overcoming problems associated with this drug, mainly the high frequency of nausea and vomiting and the high incidence of thromboembolic events [5].

The in vitro activity of these two platinum compounds has been well investigated and it is known that oxaliplatin induces more double-strandbreaks in DNA adducts compared with cisplatin, with increased cytotoxicity [6]. This could translate into a better clinical activity and possibly result in a survival benefit. Clinical phase II studies have investigated oxaliplatin-based chemotherapies for advanced gastric cancer, with results similar to those of cisplatinbased ones, and activity of these chemotherapies has also been shown in cisplatin-pretreated patients [7-11].

Differences in efficacy between oxaliplatin and cisplatin have been addressed in a big randomized study, the REAL-2, that investigated the role of capecitabine, an oral fluoropyrimidine, and oxaliplatin in three-drug regimens for the treatment of advanced gastroesophageal cancer and showed substantial survival equivalency between capecitabine and infusional 5-FU and between cisplatin and oxaliplatin [12].

Of interest, a trend to better survival with the use of capecitabine and oxaliplatin emerged and patients who were given concurrent capecitabine and oxaliplatin experienced a significantly longer survival.

Consistent with this, a recent meta-analysis of two randomized trials revealed a significant survival advantage of capecitabine compared with infusional 5-FU, but a similar analysis regarding oxaliplatin has not been conducted until now [13].

Moreover, in another trial, oxaliplatin was associated with significantly less toxicity and better tolerability, especially in the elderly [14].

The aim of the present work was to systemically review the available literature and perform a meta-analysis of randomized controlled trials (RCTs) comparing oxaliplatin to cisplatin for the treatment of advanced, unresectable esophagogastric cancer.

\section{Methods}

We searched PubMed, Cancerlit, Embase, and the Cochrane Register of Controlled Trials using various combinations of different terms "oxaliplatin", "cisplatin", "gastric cancer", and others. Results were limited to RCTs. We also looked at posters from the annual meetings of the European Society of Medical Oncology (ESMO) and the American Society of Medical Oncology (ASCO) in the past 10 years. The trial flow chart is given in Fig. 1.

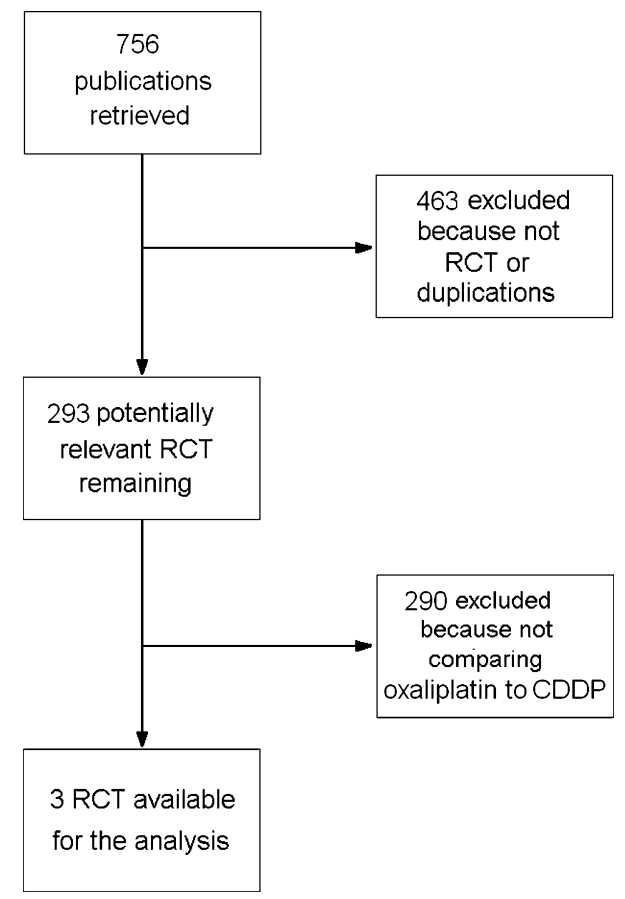

Fig. 1 Trials flow chart. $R C T$ Randomized controlled trials, $C D D P$ cisplatin

Data were retrieved from the published material. Odds ratios (ORs) were used to analyze dichotomous variables. Hazard ratios (HRs) for progression and death were combined using an inverse logarithmic conversion; $95 \%$ confidence intervals (95\% CIs) were used to determine the standard error (SE), using the formula $\mathrm{SE}=95 \% \mathrm{CI} / 1.96$. The quality of the publications was evaluated using the Jadad scale. Survival analyses were conducted using the intention-to-treat (ITT) population. Studies which allowed crossover from the control to the experimental arm were not included in the survival analysis, to avoid bias in survival times. Fixed effect, random effect, and MantelHaenszel's (M-H) method were chosen to combine and weight the individual studies. The treatment effect was reported as a point estimate with $95 \% \mathrm{CI}$. Heterogeneity was evaluated with the $Q$ test and the $I^{2}$ value. Significant heterogeneity was considered to be present for $p<0.05$ in the $Q$ test or for $I^{2}>30 \%$. The $Z$ test for overall effect and its two-sided $p$ value were assessed. Two authors independently extracted and interpreted the data. Controversies were resolved by the third author. MIX (downloaded at http://www.metaanalysis-made-easy.com/) meta-analysis and RevMan v5.0 (downloaded from http://ims.cochrane.org/revman/ download) software were used without differences in the reported outcomes. Sensitivity analyses were performed excluding one of the studies. 
Table 1 Study characteristics

\begin{tabular}{|c|c|c|c|c|}
\hline Study (references) & Patients $(N)$ & Treatment & Patient population & Jadad score \\
\hline Cunningham et al. [12] phase III & 1002 & $\begin{array}{l}\mathrm{ECF}^{\mathrm{a}}=\text { epirubicin } 60 \mathrm{mg} / \mathrm{m}^{2} \mathrm{~d} 1 \text {, cisplatin } \\
50 \mathrm{mg} / \mathrm{m}^{2} \mathrm{~d} 1,5-\mathrm{FU} \text { c.i. } 200 \mathrm{mg} / \mathrm{m}^{2} \\
\mathrm{ECX}^{\mathrm{a}}=\text { epirubicin } 50 \mathrm{mg} / \mathrm{m}^{2} \mathrm{~d} 1 \text {, cisplatin } \\
50 \mathrm{mg} / \mathrm{m}^{2} \mathrm{~d} 1 \text {, capecitabine } 625 \mathrm{mg} / \mathrm{m}^{2} \times 2 \\
\text { daily } \\
\mathrm{EOF}^{\mathrm{a}}=\text { epirubicin } 60 \mathrm{mg} / \mathrm{m}^{2} \mathrm{~d} 1 \text {, oxaliplatin } \\
85 \mathrm{mg} / \mathrm{m}^{2} \mathrm{~d} 1,5-\mathrm{FU} \text { c.i. } 200 \mathrm{mg} / \mathrm{m}^{2} \text { daily } \\
\mathrm{EOX}^{\mathrm{a}}=\text { epirubicin } 60 \mathrm{mg} / \mathrm{m}^{2} \mathrm{~d} 1, \text { oxaliplatin } \\
85 \mathrm{mg} / \mathrm{m}^{2} \mathrm{~d} 1 \text {, capecitabine } 625 \mathrm{mg} / \mathrm{m}^{2} \times 2 \\
\text { daily }\end{array}$ & $\begin{array}{l}\text { Unresectable locally } \\
\text { advanced or metastatic } \\
\text { gastric or } \\
\text { gastroesophageal junction } \\
\text { adenocarcinoma. }\end{array}$ & 3 \\
\hline Al-Batran et al. [14] Phase II & 220 & $\begin{array}{l}\text { FLO }^{\mathrm{b}}=\text { oxaliplatin } 85 \mathrm{mg} / \mathrm{m}^{2} \mathrm{~d} 1,5-\mathrm{FU} \\
2600 \mathrm{mg} / \mathrm{m}^{2} 24 \text { h-c.i. d1, FA } 200 \mathrm{mg} / \mathrm{m}^{2} \mathrm{~d} 1 \\
\text { FLP }^{\mathrm{b}}=\text { cisplatin } 50 \mathrm{mg} / \mathrm{m}^{2} \mathrm{~d} 1,5-\mathrm{FU} \\
2000 \mathrm{mg} / \mathrm{m}^{2} 24 \text { h c.i. d1, FA }=200 \mathrm{mg} / \mathrm{m}^{2} \mathrm{~d} 1\end{array}$ & $\begin{array}{l}\text { Unresectable locally } \\
\text { advanced or metastatic } \\
\text { gastric or } \\
\text { gastroesophageal junction } \\
\text { adenocarcinoma. }\end{array}$ & 2 \\
\hline Popov et al. [15] Phase II & 62 & $\begin{array}{l}\text { Arm } 1^{\mathrm{b}}=\text { oxaliplatin } 85 \mathrm{mg} / \mathrm{m}^{2} \mathrm{~d} 1,5 \text {-FU bolus } \\
400 \mathrm{mg} / \mathrm{m}^{2} \mathrm{~d} 1,2,5-\mathrm{FU} 600 \mathrm{mg} / \mathrm{m}^{2} 22 \mathrm{~h} \mathrm{c.i.} \\
\mathrm{d} 1,2, \mathrm{FA}=200 \mathrm{mg} / \mathrm{m}^{2} \mathrm{~d} 1,2 \\
\text { Arm } 2^{\mathrm{b}}=\text { cisplatin } 50 \mathrm{mg} / \mathrm{m}^{2} \mathrm{~d} 1,5-\mathrm{FU} \text { bolus } \\
400 \mathrm{mg} / \mathrm{m}^{2} \mathrm{~d} 1,2,5-\mathrm{FU} 600 \mathrm{mg} / \mathrm{m}^{2} 22 \mathrm{~h} \mathrm{c.i.} \\
\mathrm{d} 1,2, \mathrm{FA}=200 \mathrm{mg} / \mathrm{m}^{2} \mathrm{~d} 1,2\end{array}$ & $\begin{array}{l}\text { Unresectable locally } \\
\text { advanced or metastatic } \\
\text { gastric adenocarcinoma. }\end{array}$ & 2 \\
\hline
\end{tabular}

c.i. Continuous infusion, 5-FU 5-fluorouracil, $F A$ folinic acid, $N$ number of patients, $d 1,2$ day 1,2 of the cycle of chemotherapy

${ }^{a}$ Cycles repeated every 21 days

${ }^{\mathrm{b}}$ Cycles repeated every 14 days

\section{Results}

Three RCTs were available for the analysis, involving a total of 1294 patients. The results of the literature search are shown in Fig. 1. Study characteristics and Jadad scores are reported in Table 1. Patient characteristics were well balanced between the study groups. A significant reduction in the risk of death $(\mathrm{HR}=0.88,95 \%$ CI $0.78-0.99$, $p=0.04)$ and progression $(\mathrm{HR}=0.88,95 \%$ CI $0.80-0.98$, $p=0.02$ ) was observed with the use of oxaliplatin, as shown in Fig. 2. No differences were seen in responses, although there was a trend favoring oxaliplatin $(\mathrm{OR}=1.18,95 \% \mathrm{CI}=0.95-1.48, p=0.14)$. Heterogeneity was not detected and the use of random effect did not significantly alter the results. Results of toxicity analyses are shown in Table 2. Oxaliplatin was associated with significantly less all-grade and high-grade neutropenia, anemia, and alopecia, but it was also associated with increased neurotoxicity and diarrhea. Heterogeneity was not observed in any analysis, with the exception of allgrade diarrhea $(p<0.01)$. The use of random effect did not alter the results. The heterogeneity observed seemed to be related to the increased events occurring with the use of oxaliplatin in the REAL-2 study, whereas the remaining studies reported a reduced incidence of diarrhea with the use of oxaliplatin. The reasons for these differences are unknown but may be related to the schedule of concomitant chemotherapy. In one of the studies analyzed, oxaliplatin was associated with significantly higher compliance and better tolerability, especially in the older population $>65$ years. This is consistent with recent work that showed very good tolerability of an oxaliplatin combination in older patients with metastatic colorectal cancer [16].

\section{Discussion}

Despite many efforts, the prognosis of patients with advanced unresectable gastric cancer continues to be quite poor. Chemotherapy only results in palliation and a marginal survival benefit. In this regard, it is important to estimate, as exactly as possible, the toxicities of any given treatment and the absolute effects the treatment has on survival and quality of life (QoL). Many chemotherapeutic drugs and combinations have been tested in the past few decades for the treatment of advanced gastric cancer. Among them, 5-FU and cisplatin have been the most investigated and still represent the backbone of the majority of chemotherapeutic schedules used.

More recently, capecitabine and oxaliplatin have been used to replace infusional 5-FU and cisplatin, and these 
Study

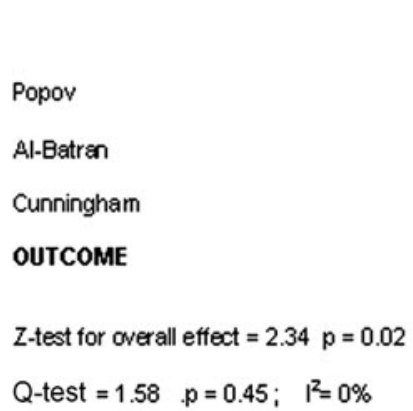

Progression-Free Survival

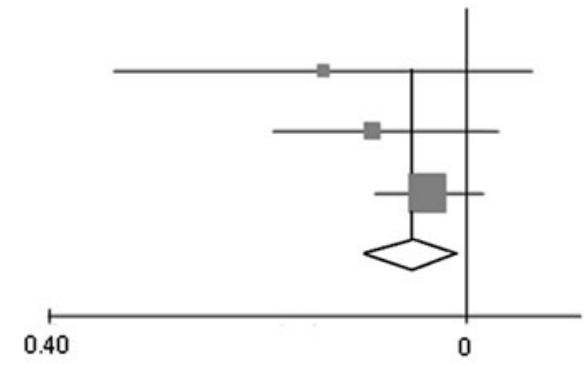

Weight

$5.00 \%$

$22.00 \%$

$73.00 \%$

$100 \%$
HR $\quad 95 \% \mathrm{Cl}$

$0.72(0.46$ to 1.15$)$

0.81 (0.66 to 1.08$)$

$0.90(0.80$ to 1.04$)$

$0.88(0.80$ to 0.98$)$

HR

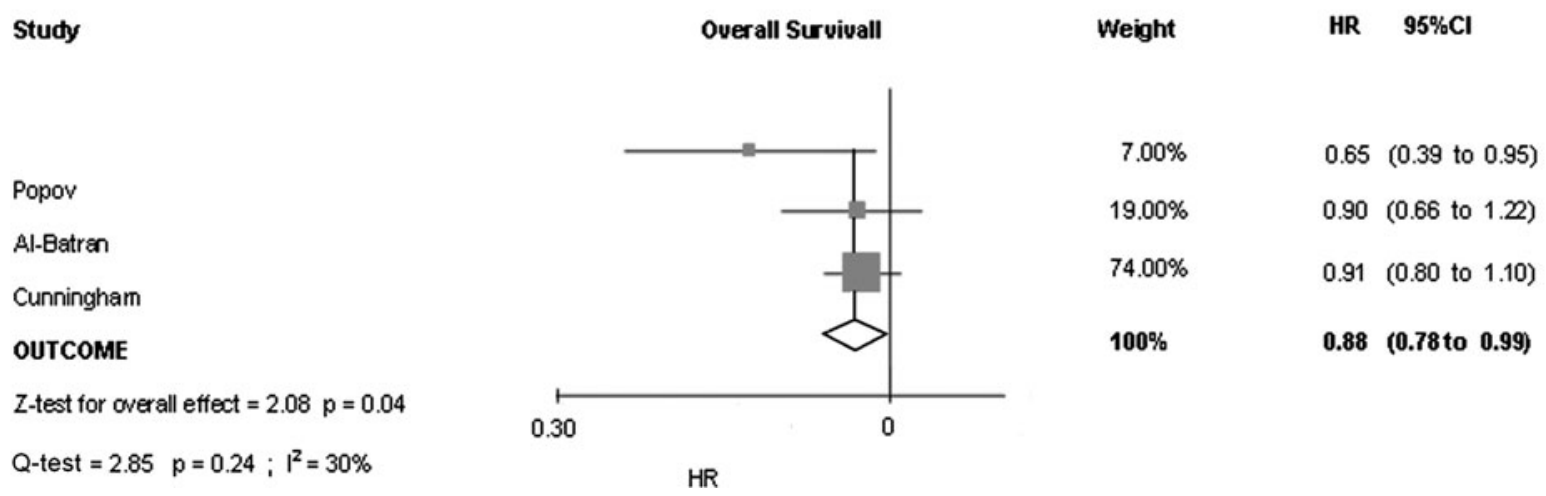

Fig. 2 Standard forest plot of the hazard ratios $(H R)$ for progression and death. Values lower than 1 favor oxaliplatin. $95 \%$ CI $95 \%$ Confidence intervals

Table 2 Toxicities

Odds ratios (ORs) were calculated from the reported events in the population assessable for toxicity $(n=1204)$. OR values of $<1$ indicate less toxicity with oxaliplatin

$C I$ confidence interval, $N A$ not assessable

\begin{tabular}{|c|c|c|c|c|}
\hline \multirow[t]{2}{*}{ Toxicity } & \multicolumn{2}{|l|}{ All grades } & \multicolumn{2}{|l|}{ Grade $3-4$} \\
\hline & OR $(95 \% \mathrm{CI})$ & $p$ value & OR $(95 \% \mathrm{CI})$ & $p$ value \\
\hline Neutropenia & $0.52(0.39-0.69)$ & $<0.01$ & $0.53(0.41-0.69)$ & $<0.01$ \\
\hline Thrombocytopenia & $1.11(0.83-1.49)$ & 0.49 & $1.05(0.62-1.83)$ & 0.85 \\
\hline Anemia & $0.36(0.28-0.48)$ & $<0.01$ & $0.64(0.41-0.98)$ & 0.04 \\
\hline Nausea/vomiting & $0.85(0.64-1.12)$ & 0.25 & $1.3(0.88-1.92)$ & 0.2 \\
\hline Diarrhea & $1.96(1.55-2.49)$ & $<0.01$ & $2.73(1.66-4.49)$ & $<0.01$ \\
\hline Stomatits & $0.84(0.66-1.08)$ & 0.18 & $0.55(0.25-1.21)$ & 0.14 \\
\hline Neurotoxicity & $8.29(6.31-10.89)$ & $<0.01$ & $6.91(3.08-15.46)$ & $<0.01$ \\
\hline Alopecia & $0.56(0.41-0.74)$ & $<0.01$ & NA & \\
\hline Thromboembolism & $0.42(0.28-0.64)$ & $<0.01$ & NA & \\
\hline
\end{tabular}

agents have been shown to possess at least equivalent efficacy to infusional 5-FU and cisplatin in RCTs.

In the big REAL-2 study these four drugs were compared, and no significant differences were observed, even though the combination of an anthracycline, capecitabine, and oxaliplatin (EOX) conferred the greatest survival benefit, with a significant increase compared with an anthracycline, cisplatin, and 5-FU regimen (ECF). A recent combined analysis confirmed that capecitabine was associated with a significant reduction in the risk of death when compared to 5-FU. We conducted the first systematic review to address whether oxaliplatin was associated with a longer survival time than cisplatin and to quantify the eventual benefit. The toxicity profiles of the two drugs were also investigated. A significant improvement in both progression-free survival and overall survival was observed with oxaliplatin, whereas no differences were evident in the analysis for responses. The absolute benefit is small and translates to a reduction in the risk of both death and progression of $12 \%$. The toxicity profiles confirmed the 
already known differences between the two drugs, with oxaliplatin being significantly associated with increased neurotoxicity, but also being significantly associated with fewer thromboembolic events and less severe neutropenia. These last two toxicities are a major cause of complications and hospitalization during chemotherapeutic treatments and represent an important threat to the patient's health, with life-threatening potential. In this regard it is important to mention that bi-weekly administration of oxaliplatin and infusional 5-FU is associated with excellent tolerability, especially in older patients, with no reported toxic deaths. Al-Batran et al. have demonstrated that this two-drug schedule is particularly well tolerated in older patients, with a significant survival benefit in this subset of patients [14]. This observation could be explained by the higher percentage of patients without treatment interruptions. Our results show that oxaliplatin reduced almost all myelotoxicities, including severe neutropenia, but increased the incidence of all-grade and severe diarrhea. However, significant inter-study heterogeneity was present, as previously mentioned. Differences in the chemotherapy regimens, possibly the presence of anthracyclines or capecitabine, could have been responsible for the increased incidence of diarrhea in the REAL-2 study in comparison to the others. It should be mentioned that in the REAL-2 study, drugs were administered on a three-weekly schedule, and this schedule could have contributed to this finding.

In our opinion the results regarding toxicities are of particular relevance. Given the small survival benefit and the frequent presence of comorbitities, and the poor performance status of patients with advanced gastric cancer, the safety profile is of critical importance to improve the QoL of patients. Only Al-Batran et al. investigated this aspect, and they found that the oxaliplatin-based regimen was associated with improved QoL, especially in older patients [14]. Severe neutropenia and thromboembolic events are life-threatening toxicities and represent an important cause of hospitalization and additional treatments in patients receiving chemotherapy. Both types of events increase the indirect costs of a treatment, often representing an important percentage of the total expense. Although a formal cost-effectiveness analysis was not performed, our results indicate that oxaliplatin could be associated with improved cost-effectiveness through increased patient survival and reduction of the indirect costs. Moreover, patients receiving oxaliplatin do not need prehydration and the drug is easier to administer in a routine clinical setting. We recognize that the present analysis has some limitations. First, only three studies have been conducted until now, with one trial including the majority of the patients. Another possible remark is regarding the use of aggregate data instead of individual patient data (IPD). The use of IPD has some advantage over the use of aggregatepatient data (APD), but the use of IPD needs considerable time, resources, and worldwide collaborations. Moreover, IPD are not available to the scientific community and can only be obtained at the discretion of the principal investigators. APD are based on published materials and can easily be repeated by anyone. APD-based and IPD-based metaanalyses have been shown to return almost identical results, so it is unlikely that the use of IPD would have significantly altered our results [17, 18].

Another limitation is that second-line treatments were not reported, so it was not possible to consider their possible impact on survival. Although the role of second-line treatments has been a matter of debate, a recent report from Thuss-Patience et al. [19] showed improved survival with the second-line administration of irinotecan over best supportive care. However, second-line treatments obviously do not alter the progression-free survival. Finally, publication bias could not be addressed because of the small number of studies, so it is not possible to rule out that non-published negative studies exist.

In recent years, newer biological agents such as the tyrosine kinase inhibitors (TKIs) and monoclonal antibodies (MoAbs) directed to critical tumoral targets such as the epidermal growth factor receptor (EGFR), human epidermal growth factor receptor 2 (HER-2), and vascular endothelial growth factor (VEGF), have been investigated in many solid malignancies, including gastric cancer. Unfortunately, with the exception of trastuzumab, an HER2 specific monoclonal antibody which was shown to improve survival in HER-2+ gastric and esophagogastric adenocarcinoma, none of these agents has been demonstrated to improve survival in comparison with chemotherapy [20]. Future studies should focus on better regimens to be obtained with combinations of chemotherapeutic agents and these biological agents. In conclusion, the present analysis has confirmed that oxaliplatin is significantly less toxic than cisplatin, with the exception of diarrhea, which seems, however, to be a schedule-dependent toxicity, related to a three-drug, three-weekly administration. Our results support the existence of a small but significant benefit in both progression-free and overall survival associated with the use of oxaliplatin.

Conflict of interest All the authors declare no conflict of interest. The work has been entirely funded by the Azienda Unità Sanitaria Locale 11 (AUSL 11).

\section{References}

1. Parkin DM, Bray F, Ferlay J, Pisani P. Global cancer statistics, 2002. CA Cancer J Clin. 2005;55:74-108.

2. Valentini V, Cellini F, Minsky BD, Mattiucci GC, Balducci M, D'Agostino G, et al. Survival after radiotherapy in gastric cancer: systematic review and meta-analysis. Radiother Oncol. 2009; 92(2):176-83. 
3. Cunningham D, Allum WH, Stenning SP, Thompson JN, Van de Velde CJ, Nicolson M, et al. Perioperative chemotherapy versus surgery alone for resectable gastroesophageal cancer. N Engl J Med. 2006;355(1):11-20.

4. Wagner AD, Groethe W, Haerting J, Kleber G, Grothey A, Fleig WE. Chemotherapy in advanced gastric cancer: a systematic review and meta-analysis based on aggregate data. J Clin Oncol. 2006;24:2903-9.

5. Starling N, Rao S, Cunningham D, Iveson T, Nicolson M, Coxon $\mathrm{F}$, et al. Thromboembolism in patients with advanced gastroesophageal cancer treated with anthracycline, platinum, and fluoropyrimidine combination chemotherapy: a report from the UK National Cancer Research Institute Upper Gastrointestinal Studies Group. J Clin Oncol. 2009;27(23):3786-93.

6. Chaney SG, Campbell SL, Bassett E, Wu Y. Recognition and processing of cisplatin- and oxaliplatin-DNA adducts. Crit Rev Oncol Hematol. 2005;53(1):3-11.

7. Kim DY, Kim JH, Lee SH, Kim TY, Heo DS, Bang Y, et al. Phase II study of oxaliplatin, 5-fluorouracil and leucovorin in previously platinum-treated patients with advanced gastric cancer. Ann Oncol. 2003;14(3):383-7.

8. Louvet C, André T, Tigaud JM, Gamelin E, Douillard JY, Brunet $\mathrm{R}$, et al. Phase II study of oxaliplatin, fluorouracil and folinic acid in locally advanced or metastatic gastric cancer patients. J Clin Oncol. 2002;20(23):4543-8.

9. Al-Batran SE, Atmaca A, Jaeger E et al. Phase II trial of biweekly infusional fluorouracil, folinic acid and oxaliplatin in patients with advanced gastric cancer. J Clin Oncol. 2004;22(4):685-63.

10. Souglakos J, Syrigos K, Potamianou A, Polyzos A, Boukovinas I, Androulakis N, et al. Combination of irinotecan (CPT-11) plus oxaliplatin (L-OHP) as first-line treatment in locally advanced or metastatic gastric cancer: a multicentre phase II trial. Ann Oncol. 2004;15(8):1204-9.

11. Correale P, Fulfaro F, Marsili S, Cicero G, Bajardi E, Intrivici C, et al. Gemcitabine plus oxaliplatin, folinic acid and 5-fluorouracil (FOLFOX-4) in patients with advanced gastric cancer. Cancer Chemother Pharmacol. 2005;56(6):563-8.

12. Cunningham D, Starling N, Rao S, Iveson T, Nicolson M, Coxon F, et al. Upper Gastrointestinal Clinical Studies Group of the National Cancer Research Institute of the United Kingdom. Capecitabine and oxaliplatin for advanced gastric cancer. N Engl J Med. 2008;358:36-46.
13. Okines AFC, Norman AR, McCloud P, Kang YK, Cunningham D. Meta-analysis of the REAL-2 and ML17032 trials: evaluating capecitabine-based combination chemotherapy and infused 5 -fluorouracil-based combination chemotherapy for the treatment of advanced oesophagogastric cancer. Ann Oncol. 2009;20: 1529-34.

14. Al-Batran SE, Hartmann JT, Probst S, Schmalenberg H, Hollerbach S, Hofheinz R, et al. Phase III trial in metastatic gastroesophageal adenocarcinoma with fluorouracil, leucovorin plus either oxaliplatin or cisplatin: a study of the Arbeitsgemeinschaft Internistische Onkologie. J Clin Oncol. 2008;26(9):1435-42.

15. Popov I, Radosevic-Jelic L, Jezdic S, Milovic M, Borojevic N, Stojanovic S, et al. Biweekly oxaliplatin, fluorouracil and leucovorin versus cisplatin, fluorouracil and leucovorin in patients with advanced gastric cancer. J BUON. 2008;13:505-11.

16. Goldberg RM, Tabah-Fisch I, Bleiberg H, de Gramont A, Tournigand C, Andre T, Rothenberg ML, Green E, Sargent DJ. Pooled analysis of safety and efficacy of oxaliplatin plus fluorouracil/ leucovorin administered bimonthly in elderly patients with colorectal cancer. J Clin Oncol. 2006;24:4085-91.

17. Bria E, Gralla RJ, Raftopoulos H, Giannarelli D. Comparing two methods of meta-analysis in clinical research-individual patient data-based (IPD) and literature-based abstracted data (AD) methods: analyzing five oncology issues involving more than 10,000 patients in randomized clinical trials (RCTs). J Clin Oncol. 2007;25(18S) (Suppl Abstr 6512).

18. Lyman GH, Kurderer NM. The strengths and limitations of metaanalyses based on aggregate data. BMC Med Res Methodol. 2005;5:14.

19. Thuss-Patience PC, Kretzschmar A, Deist T, Hinke A, Bichev D, Lebedinzew B, Schumacher G. Irinotecan versus best supportive care (BSC) as second-line therapy in gastric cancer: a randomized phase III study of the Arbeitsgemeinschaft Internistische Onkologie (AIO). J Clin Oncol. 2009;27(15 s) (Suppl Abstr 4540).

20. Van Cutsem E, Kang Y, Chung H, Shen L, Sawaki A, Lordick F, et al. Efficacy results from the ToGA trial: a phase III study of trastuzumab added to standard chemotherapy (CT) in first-line human epidermal growth factor receptor 2 (HER2)-positive advanced gastric cancer (GC). J Clin Oncol. 2009;27(18 s):(Suppl abstr LBA4509). 